\title{
Berry's Phase Magnification in Microcoil Resonators
}

\author{
Timothy Lee, Neil G. R. Broderick and Gilberto Brambilla \\ Optoelectronics Research Centre, University of Southampton, Southampton, \\ Hampshire, SO17 1BJ, United Kingdom \\ tl305@orc.soton.ac.uk
}

Research towards understanding the topological Berry's phase $\left(\phi_{B}\right)$ has been driven by its manifestation in various interesting quantum mechanical and optical phenomena. In helical fibre structures, the generation of $\phi_{B}$ is well documented [1,2], and recently the amplification of Berry's phase using a ring resonator was observed [3]. In this work, we show it is theoretically possible to generate a large Berry's phase using compact optical microcoil resonators (OMRs) formed by tightly winding a microfibre around a rod such that light can evanescently couple between neighbouring turns. In particular, the effect of $\phi_{B}$ on the OMR transmission is analysed, in order to understand the associated polarisation effects which are potentially important for developing OMR applications.

In a fibre helix with a linearly polarised input light, $\phi_{B}$ can be modelled classically as a rotation of the polarisation plane according to the helix-torsion $(\tau)$ [1]. If $A_{j}^{x}$ and $A_{j}^{y}$ denote the field amplitudes along the normal and binormal axes respectively in the $j^{\text {th }}$ turn of the coil as in Fig. 1(a), the OMR coupled mode equations [4,5] are modified as follows:

$$
\begin{aligned}
\frac{d A_{j}^{x}}{d s} & =i \kappa_{x}\left(A_{j-1}^{x}+A_{j+1}^{x}\right)+\tau A_{j}^{y}+\left(i \frac{C^{2}}{2 \beta}-\alpha\right) A_{j}^{x} \\
\frac{d A_{j}^{y}}{d s} & =i \kappa_{y}\left(A_{j-1}^{y}+A_{j+1}^{y}\right)-\tau A_{j}^{x}-\alpha A_{j}^{y}
\end{aligned}
$$

where $\kappa_{x, y}$ are the coupling coefficients between two $x$ or two $y$ polarised modes in adjacent turns, $\alpha$ is the loss coefficient, and the curvature term $(C)$ accounts for the bend induced propagation mismatch. Note that $A^{x}$ and $A^{y}$ would be uncoupled if the Berry's phase term were neglected $(\tau=0)$, and hence Berry phase effects are clearly seen in the coupling of light from one orthogonal state of polarisation to another.

Solving these equations with an initially $x$ polarised input shows that, on resonance, a substantial fraction $\eta$ of the power can be coupled into the $y$ polarisation at the output due to the recirculation of light in the OMR, e.g. $\eta=0.66$ in Fig. 1(b). Compared to an uncoupled helix $\left(\kappa_{x, y}=0\right)$ in which the light would only make a single pass through the coil, $\eta$ can be up to $10^{2}-10^{3}$ times larger. Off-resonance, $\eta$ falls to $10^{-3}$ since the light is simply coupled up the turns and out of the OMR.

As $\kappa_{x, y}$ depend exponentially on the pitch $p$ between the turns, even small variations in $p$ can noticeably alter $\eta$. Fig.1(c) illustrates this for a range of $p$ over which $4000 \mathrm{~m}^{-1}<\kappa_{\mathrm{x}, \mathrm{y}}<6000 \mathrm{~m}^{-1}$ : when critically coupled, $\eta$ is close to $100 \%$, whilst for other values of $p$ the resonance has a low $\mathrm{Q}$ factor and thus $\eta \approx 0$.

Decreasing the rod radius to increase torsion and using a smaller core diameter in conjunction with a larger pitch would also maximise the generation of Berry's phase. Practically, with an appropriate pitch the effects of Berry's phase on the output of an OMR are sufficiently large to be detected experimentally.
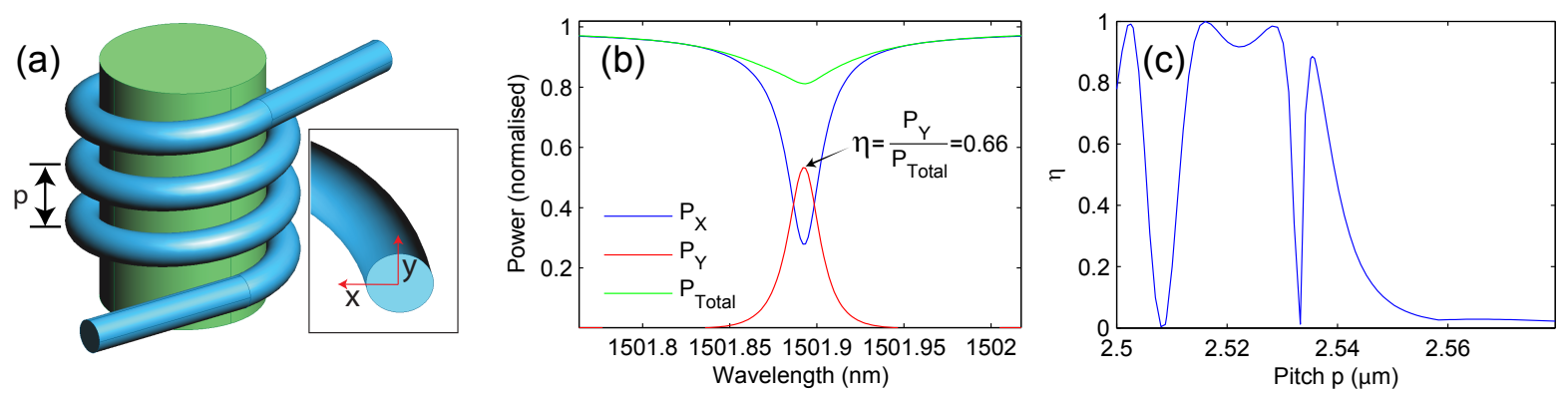

Fig. 1. (a) Microcoil structure. (b) Typical output spectrum of a resonance . (c) Fraction of output power in $y$ polarisation $\eta$ vs pitch $p$. In all graphs, the input is $\left[A_{x}, A_{y}\right]=[1,0]$, core diameter $=1 \mu \mathrm{m}$, OMR has 3 turns, and rod diameter $=0.2 \mathrm{~mm}$.

\section{References}

[1] M. V. Berry, "Interpreting the anholonomy of coiled light," Nature 326, 277-278 (1987).

[2] A. Tomita, R. Y. Chiao, “Observation of Berry's Topological Phase by Use of an Optical Fiber,” Phys. Rev. Lett. 57, 937-940 (1986).

[3] I. Golub, T. Audet, L. Imobekhai, “Observation of Berry's phase amplification by a ring resonator," J. Opt. Soc. Am. B 27, 1698-1700 (2010).

[4] M. Sumetsky, "Optical fiber microcoil resonators," Opt. Expr. 12, 2303-2316 (2004).

[5] N. G. Broderick, “Optical Snakes and Ladders: Dispersion and nonlinearity in microcoil resonators,” Opt. Expr. 16, 16247-16254 (2008). 